\title{
Theoretical and laboratory spectra of sodium perturbed by molecular hydrogen ${ }^{\star}$
}

\author{
N. F. Allard ${ }^{1,2}$, J. F. Kielkopf ${ }^{3}$, F. Spiegelman ${ }^{4}$, G. Tinetti ${ }^{5}$, and J. P. Beaulieu ${ }^{2,5}$ \\ ${ }^{1}$ GEPI, Observatoire de Paris, UMR 8111, CNRS, Université Paris VII, 61 avenue de l'Observatoire, 75014 Paris, France \\ e-mail: nicole.allard@obspm. fr \\ 2 Institut d'Astrophysique de Paris, UMR 7095, CNRS, Université Paris VI, 75014 Paris, France \\ 3 Department of Physics and Astronomy, University of Louisville, Louisville, KY 40292, USA \\ ${ }^{4}$ Laboratoire de Chimie et Physique Quantique, UMR 5626, CNRS, Université Paul Sabatier, 118 route de Narbonne, \\ 31400 Toulouse, France \\ 5 Department of Physics and Astronomy, University College London, Gower Street, WC1E6BT London, UK
}

Received 14 February 2012 / Accepted 17 May 2012

\begin{abstract}
We present new unified theory line profiles of neutral Na perturbed by $\mathrm{H}_{2}$. We used a priori $\mathrm{Na}-\mathrm{H}_{2}$ potentials, transition dipole moments and validated pseudo-potentials as input to the line shape, and evaluated the profiles for temperatures and densities appropriate for modeling exoplanet and brown dwarf atmospheres. The theory for the resonance lines was compared with new laboratory spectra of sodium to test the validity of the potentials and resulting profiles. The Lorentzian function commonly used to approximate a collisional line profile in radiative transfer calculations is shown to be inadequate, except within a few halfwidths of the line center. In the far wing, the opacity caused by collisions may be several orders of magnitude greater than the extrapolation of the Lorentzian core.
\end{abstract}

Key words. atomic data - line: profiles - brown dwarfs - planets and satellites: atmospheres

\section{Introduction}

The construction of model atmospheres and synthetic spectra for cool brown dwarfs and extrasolar giant planets is necessary to derive reliable atmospheric parameters and the surface chemical composition for these objects. The studies of observed $\mathrm{L}$ and T dwarfs by Liebert et al. (2000) and Burrows et al. (2001) clearly showed the importance of the extended wings of both sodium and potassium doublets, and they pointed out the need for line profile calculations more accurate than Lorentzian profiles. Clearly understanding the shape of these lines is essential to modeling the transport of radiation from the interior.

A first improvement was achieved by Burrows \& Volobuyev (2003) using multiconfiguration self-consistent field Hartree-Fock potentials in the Szudy theory (Szudy \& Baylis 1975, 1996). In Allard et al. (2003) we presented absorption profiles of sodium and potassium perturbed by helium and molecular hydrogen. The line profiles were calculated in a unified line shape semi-classical theory (Allard et al. 1999) using Rossi $\&$ Pascale (1985, hereafter RP85) pseudo-potentials. The pressure broadening calculations were extended to the case of the other alkalies: lithium, rubidium, and cesium, where calculations were performed for the $D 1$ and $D 2$ lines from $T_{\text {eff }}=500 \mathrm{~K}$ to $3000 \mathrm{~K}$ with a step size of $500 \mathrm{~K}$ (Allard et al. 2005; Allard $\&$ Spiegelman 2006). Tables of absorption coefficients are now provided in an on-line database for convenient use in atmosphere modeling ${ }^{1}$.

\footnotetext{
^ Line profile data are only available at the CDS via anonymous ftp to cdsarc.u-strasbg.fr (130.79.128.5) or via

http://cdsarc.u-strasbg.fr/viz-bin/qcat?]/A+A/543/A159

1 http://mygepi.obspm.fr/ allard/
}

Key observations with Spitzer and Hubble space telescopes and from the ground have recently given us real insights into the conditions and composition of the atmospheres of some of the most unusual exoplanets so far discovered, the class known as hot-Jupiters (Tinetti et al. 2007; Swain et al. 2008; Snellen et al. 2010; Swain et al. 2010; Jensen et al. 2011; Waldmann et al. 2012). Sodium was first detected in the atmosphere of HD 209458b (Charbonneau et al. 2002). Observations of other planets have been obtained with ground-based and HST spectroscopy in the optical (Redfield et al. 2008; Snellen et al. 2008; Jensen et al. 2011), confirming that alkali metals are present in hot-Jupiter atmospheres. Désert et al. (2008) presented a low-resolution STIS spectrum from HST covering the range from 4000 to $8000 \AA$. They adopted a Lorentzian profile for the alkali atoms following Burrows et al. (2000) with a temperature profile from Sing et al. (2008). Désert and collaborators show in Fig. 1 that the $\mathrm{Na}$ I line is decently fitted in the blue wing. However, they noted a strong discrepancy in the red wing from $6200 \AA$ onward, between the observed absorption coefficient and a prediction based on a Lorentzian core. They interpreted this as a result of additional absorption due to $\mathrm{TiO}$ and VO molecules in the atmosphere of HD 209458b. We will show, however, that profiles of the far wing of the Na resonance lines may have orders of magnitude more absorption than a Lorentzian. As a consequence, it appears that when the far wing absorption due to alkali atoms in a dense $\mathrm{H}_{2}$ atmosphere is significant, exact atomic lineshape physics should be incorporated into spectral models.

$\mathrm{Ab}$ initio calculations of the potentials of $\mathrm{K}-\mathrm{H}_{2}$ (hereafter S06) were computed by one of us (FS). They have been reported in Allard et al. (2007c) and are now extended to $\mathrm{Na}-\mathrm{H}_{2}$ 
(hereafter S11). In Sect. 2 we describe new calculations of the $\mathrm{Na}-\mathrm{H}_{2}$ potential energy surfaces in which the two electrons of $\mathrm{H}_{2}$ and the valence electron of sodium are explicit.

We present new theoretical $\mathrm{NaH}_{2}$ line profiles for the $3 s-3 p$ transition in Sect. 3. In a continuation of the work presented recently (Allard 2011a,b; Allard et al. 2012), we highlight the regions of interest near the $\mathrm{Na}-\mathrm{H}_{2}$ and $\mathrm{K}-\mathrm{H}_{2}$ quasimolecular satellites for comparison with previous results described in Allard et al. (2007c). We describe the experimental work in Sect. 4 and compare theoretical and laboratory spectra in Sect. 5.

\section{2. $\mathrm{Na}-\mathrm{H}_{2}$ diatomic potentials without spin-orbit coupling}

The interactions of an alkali atom with $\mathrm{H}_{2}$ depend on the orientation of the $\mathrm{H}_{2}$ axis with the collision axis. In the work of Rossi $\&$ Pascale (1985), the alkali- $\mathrm{H}_{2}$ molecular potentials were calculated using model potentials completed by polarization operators for the two symmetries $C_{2 v}\left(\Theta=90^{\circ}\right)$ and $C_{\infty v}(\Theta=0)$, where $\Theta$ is the angle between the direction of the molecular axis and the interaction axis. In these calculations, the alkali- $\mathrm{H}_{2}$ complex was considered as a system with a single active electron.

In the present work, we achieved similar calculations for $\mathrm{Na}-\mathrm{H}_{2}$ as we found for $\mathrm{K}-\mathrm{H}_{2}$ in a previous publication (Allard et al. 2007c). Namely, the two electrons of $\mathrm{H}_{2}$ are explicit, the sodium atom is described by a valence pseudopotential, complemented by a core-polarization operator to account for polarization and core polarization effects (Müller et al. 1984). The Gaussian basis set at each hydrogen center was 7s6p4d and that at sodium was $7 \mathrm{~s} 5 \mathrm{p} 6 \mathrm{~d}$, including diffuse Gaussian functions. The smallest exponents are $\zeta=0.0030,0.0058$ and 0.0015 for $\mathrm{s}, \mathrm{p}$, and d Gaussian type orbitals. The core dipole polarisability of sodium $\left(\mathrm{Na}^{+}\right)$was taken as $\alpha=0.985 a_{0}^{3}$, and a step-like cutoff function with $\rho=1.39 a_{0}$ was used (Foucrault et al. 1992). This provides atomic transition energies $v_{3 \mathrm{~s}-3 \mathrm{p}}=16951 \mathrm{~cm}^{-1}$ (experimental spin-orbitless value $16968 \mathrm{~cm}^{-1}$ ), and $v_{3 \mathrm{~s}-4 \mathrm{~s}}=$ $25779 \mathrm{~cm}^{-1}$ (experimental value $25740 \mathrm{~cm}^{-1}$ ). For $\mathrm{Na}-\mathrm{H}_{2}$, quasi-full three-electron configuration interaction (CI) was achieved using the MRCI/QDPT2 CIPSI package of Toulouse (Huron et al. 1973; Spiegelmann \& Malrieu 1984). First eigenfunctions were obtained within the subspace of all single and double excitations (SDCI). All triply excited determinants having a perturbative contribution in the wavefunction larger than $\tau=10^{-5}$ were added in the final variational CI. The total perturbative contribution of the remaining triple excitations in the energy for all states investigated was less than $10^{-6}$ Hartree, whatever the state and geometry. The transition dipole moments were computed using the variational wavefunctions of the final CI incorporating the core polarisation potential contributions. The ab initio calculations of the potentials were carried out for the $\mathrm{C}_{2 v}$ (T-shape) symmetry group and the $\mathrm{C}_{\infty v}$ (linear) symmetry group in a wide range of distances $R$ between the Na atom and the center-of-mass of the molecule $\mathrm{H}_{2}$. In the calculation of the complex, the bond length of $\mathrm{H}_{2}$ was kept fixed at the equilibrium value $r_{\mathrm{e}}=1.401 \mathrm{au}$. The quality of the calculation on the $\mathrm{H}_{2}$ subsystem is the same as that in our previous work on $\mathrm{K}-\mathrm{H}_{2}$. The full CI dissociation energy is $D_{\mathrm{e}}=38119 \mathrm{~cm}^{-1}$ at $R_{\mathrm{e}}=1.401 a_{0}$.

In $\mathrm{C}_{2 v}$ symmetry $\left(\Theta=90^{\circ}\right)$, the ground state belongs to the ${ }^{2} \mathrm{~A}_{1}$ manifold, the excited states to the ${ }^{2} \mathrm{~B}_{1}$ and ${ }^{2} \mathrm{~B}_{2}$ and ${ }^{2} \mathrm{~A}_{1}$ manifolds respectively. In $\mathrm{C}_{\infty v}$ symmetry, the ground state $\mathrm{X}$ has ${ }^{2} \Sigma^{+}$symmetry, while the $3 p$ excited configuration generates

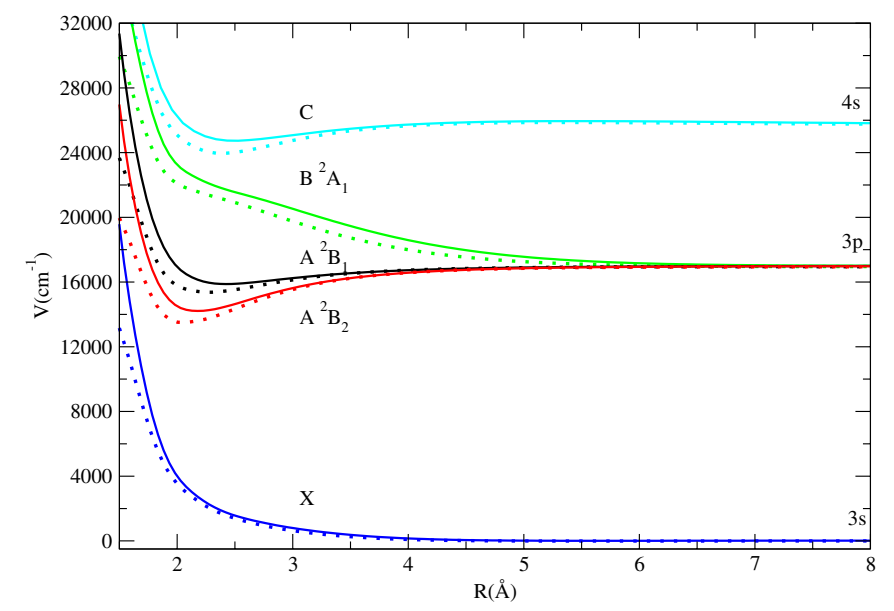

Fig. 1. Potential curves for the $3 s, 3 p$ and $4 s$ states of $\mathrm{NaH}_{2}$ molecule without spin-orbit coupling for the $\mathrm{C}_{2 v}$ symmetry of $\mathrm{S} 11$ (dotted lines) compared to RP85 (full line).

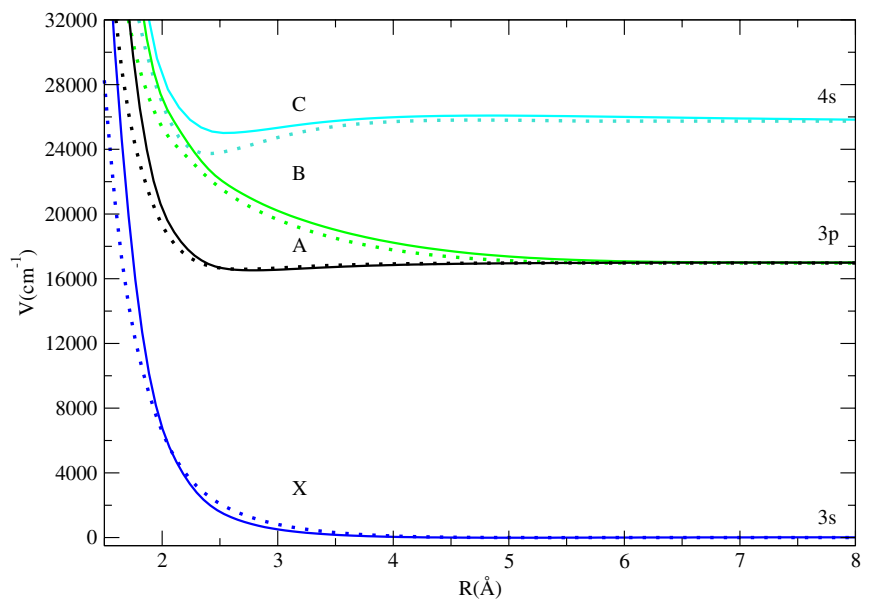

Fig. 2. Potential curves for the $3 \mathrm{~s}, 3 \mathrm{p}$ and $4 \mathrm{~s}$ states of $\mathrm{NaH}_{2}$ molecule without spin-orbit coupling for the $\mathrm{C}_{\infty v v}$ symmetry of $\mathrm{S} 11$ (dotted lines) compared to RP85 (full line).

one two-fold ${ }^{2} \Pi$ state (A, with attractive character) and one essentially repulsive ${ }^{2} \Sigma^{+}$state (B).

Figures 1 and 2 present the potential energy surfaces $V_{\mathrm{e}}(R)=$ $E_{\mathrm{e}}(R)-E_{3 \mathrm{~s}}^{\infty}$ in the $\mathrm{C}_{2 v}$ and the $\mathrm{C}_{\infty v}$ symmetries, respectively. Obviously, the major difference with respect to RP85 is that the resulting ab initio potentials are systematically less repulsive than theirs. This is certainly owing to a better account here of the repulsion between the valence electron of $\mathrm{Na}$ and the electrons of $\mathrm{H}_{2}$ explicitly involved here.

\section{Study of the far wings and quasi-molecular $\mathrm{Na}-\mathrm{H}_{2}$ line satellites for the $3 s-3 p$ and $3 p-4 s$ transitions}

Collision-broadened lines show features that arise from the changed structure of the radiating atom in the presence of another atom. As such, their presence is sensitive to the conditions of the radiating system and reveal the composition, temperature, and pressure of the source.

\subsection{Blue wings}

Blue satellites on the line are predicted in the far wing of alkali-He/ $\mathrm{H}_{2}$ line profiles. These satellite bands of alkali metals 
N. F. Allard et al.: Na broadened by $\mathrm{H}_{2}$

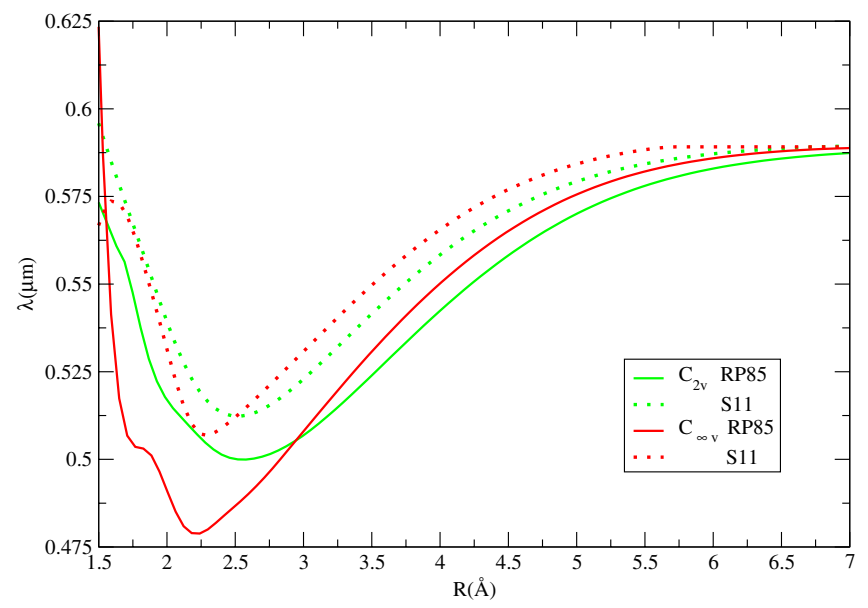

Fig. 3. Wavelengths corresponding to difference potentials $\Delta V$ for the $\mathrm{B}-\mathrm{X}$ states of $\mathrm{NaH}_{2}$ (symmetry $\mathrm{C}_{2 v}$ and $\mathrm{C}_{\infty v}$ ).

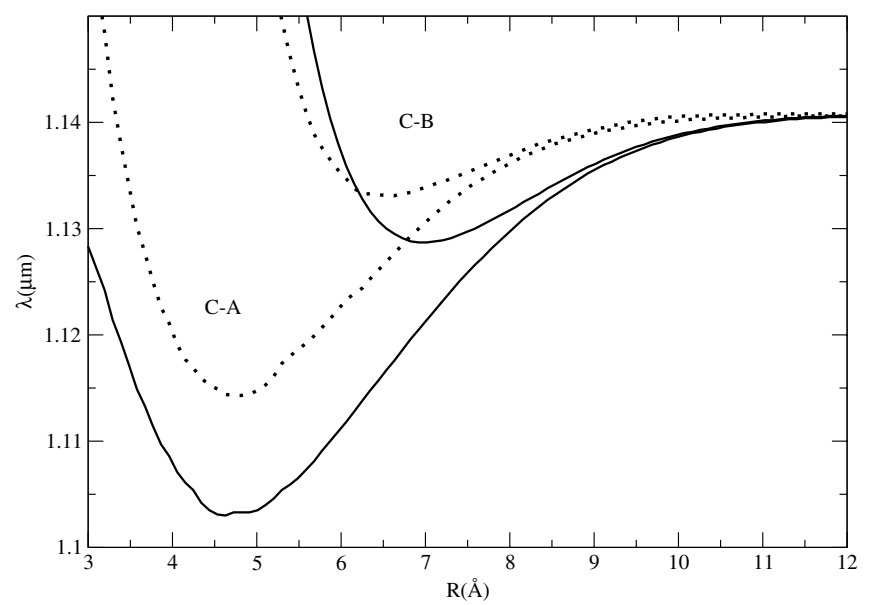

Fig. 4. Wavelengths corresponding to difference potentials $\Delta V$ for the $3 \mathrm{pP}_{3 / 2}-4 \mathrm{~s}$ transition of $\mathrm{NaH}_{2}$ (symmetry $\mathrm{C}_{2 v}$ ) using RP85 (full line) compared to $\mathrm{S} 11$ (dotted lines).

perturbed by rare gas have been extensively studied in the past (Allard \& Kielkopf 1982, and references therein). There was a renewal of interest (Burrows \& Volobuyev 2003; Allard et al. 2003, 2005, 2007c; Allard \& Spiegelman 2006; Zhu et al. 2005, 2006; Alioua \& Bouledroua 2006; Alioua et al. 2008) because alkali-metals are a prominent source of opacity in the spectra of brown dwarfs (Allard et al. 2003, 2007a). Blue satellite bands in alkali-He/ $\mathrm{H}_{2}$ profiles can be predicted from the extrema in the difference potentials $\Delta V(R)$, given by

$\Delta V(R) \equiv V_{\mathrm{e}^{\prime} \mathrm{e}}[R(t)]=V_{\mathrm{e}^{\prime}}[R(t)]-V_{\mathrm{e}}[R(t)]$,

which represents the difference between the electronic energies of the quasi-molecular $\mathrm{e}^{\prime}-\mathrm{e}$ transition. The wavelength $\lambda(R)$ corresponding to the $\mathrm{B}-\mathrm{X}$ transition and $\mathrm{C}-\mathrm{A} / \mathrm{B}$ transitions is shown in Figs. 3 and 4.

The differences on the $B$ state shown in Figs. 1-3 affect the blue B - X satellite position. The line satellite is at $0.515 \mu \mathrm{m}$ for $\mathrm{S} 11$ and at $0.5 \mu \mathrm{m}$ for RP85. The extension of the blue wing of the $3 p-4$ s transition is also reduced for S11 and the red wing is unchanged, as seen in Fig. 6.

Absorption cross-sections for $3 p-3 d, 3 p-4 s$, and $4 s-4 p$ transitions in the infrared are shown in Fig. 4 of Allard et al. (2012).

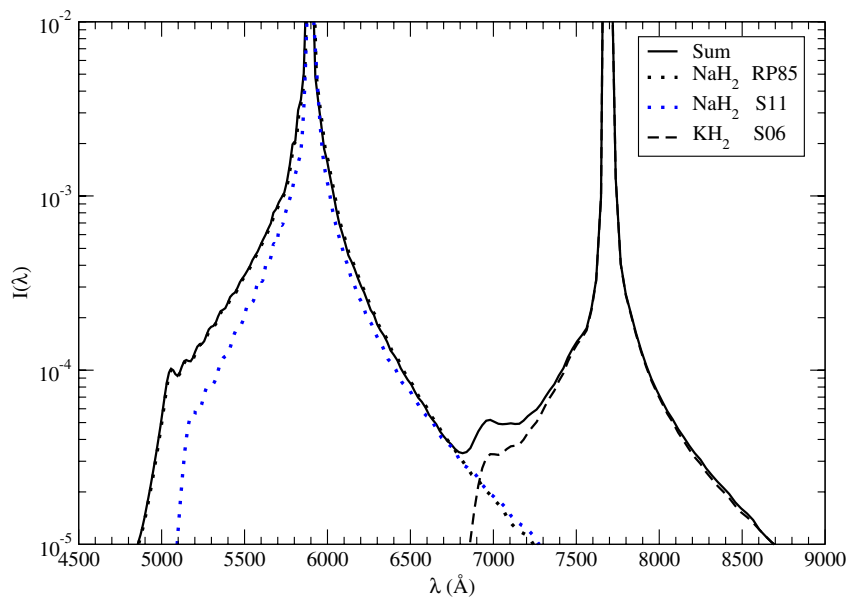

Fig. 5. Sum of Na and K alkali profiles perturbed by molecular hydrogen, weighted by their line strength at solar abundance compared to the contribution of $\mathrm{Na}$ and $\mathrm{K}$ for a fixed molecular hydrogen density $\left(n_{\mathrm{H}_{2}}=10^{19} \mathrm{~cm}^{-3}\right.$ at $\left.1500 \mathrm{~K}\right)$.

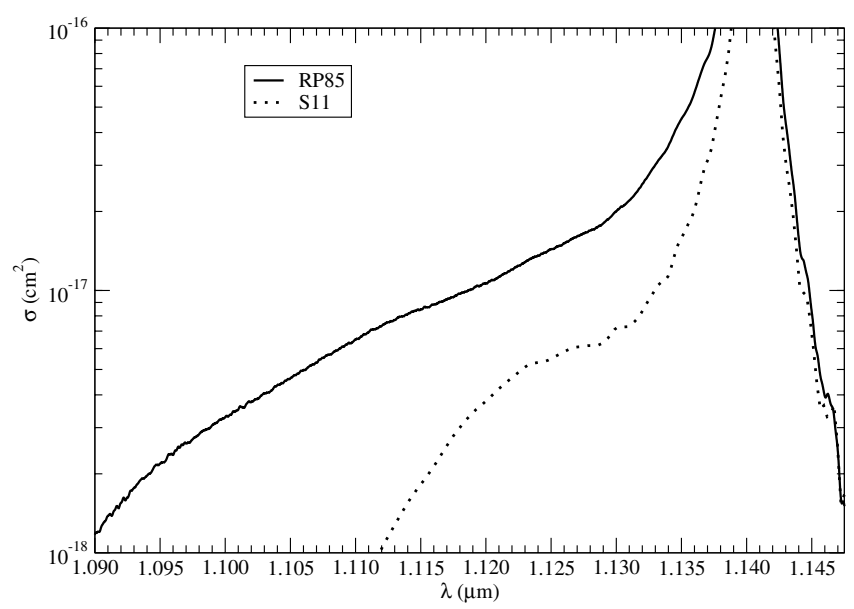

Fig. 6. Absorption cross sections of the $3 \mathrm{pP}_{3 / 2} 4 \mathrm{~s}$-line component of $\mathrm{NaH}_{2}$, taking into account the two symmetries $\mathrm{C}_{2 v}$ and $\mathrm{C}_{\infty v}$ using RP85 (full line) compared to S11 (dotted lines) $\left(n_{\mathrm{H}_{2}}=10^{19} \mathrm{~cm}^{-3}\right.$ at $1000 \mathrm{~K})$.

\subsection{Red wings}

While B states radiate in the blue wing, A states radiate in the red wing. The total resonance line profiles resulting from both $\mathrm{Na}$ and $\mathrm{K}$ perturbed by collisions with molecular hydrogen is shown in Fig. 5. The sum was calculated according to Eq. (51) of Allard et al. (1999). Each alkali profile was then weighted by its line strength. We can clearly see the contribution of each alkali in the region of the $\mathrm{K}-\mathrm{H}_{2}$ line satellite. The overlap of the two wings results in an enhancement of the contrast of the $\mathrm{K}-\mathrm{H}_{2}$ feature in the line wing. We notice in Fig. 5 that the $\mathrm{Na}-\mathrm{H}_{2}$ red wing is almost unchanged, leading to a $\mathrm{K}-\mathrm{H}_{2}$ line satellite at the same position as predicted in Allard et al. (2007c).

\section{Experimental determination of the $\mathrm{Na} 3 s-3 p$ absorption transition broadened by collisions with $\mathrm{H}_{2}$}

Collisions with $\mathrm{H}_{2}$ are preponderant in brown dwarf atmospheres at an effective temperature of about $1000 \mathrm{~K}$, and comparable temperatures can be reached in the laboratory. 
The absorption spectrum of $\mathrm{Na}$ in an $\mathrm{H}_{2}$ buffer gas was measured in a series of experiments designed to provide a relative absorption coefficient primarily for the blue (short wavelength) wing of the $3 s-3 p$ doublet. The measurements, which were based on conventional absorption spectroscopy, used a methodology that has been described previously (Kielkopf 1980, 1983). In this section we will briefly describe the experiment design, the spectroscopic instruments used to measure the spectrum, and the reduction of the data to useful absorption coefficients. The obtained spectra are compared with the theoretical spectra in the following section.

The stainless-steel absorption cell used in this work was $76 \mathrm{~cm}$ long and had a $2.2 \mathrm{~cm}$ inside diameter. It was heated in a well-defined $30 \mathrm{~cm}$ long central section, and closed with flat, highly polished, fused silica windows using O-ring sealed highvacuum fittings. The cell was attached to a high-vacuum system with a cold trap, diffusion, and rough pumps, and diagnostic pressure gauges that ensured a leak-free system. Buffer gases were metered into the cell through a stainless-steel manifold. After evacuation, purging, and filling, the cell's operating pressure was determined with a capacitive manometer to an accuracy of \pm 1 Torr.

At the beginning of a run, $99.95 \%$-pure $\mathrm{Na}$ in a glass ampoule was introduced into the center of an open-ended cell under flowing He. The cell was closed by attaching its windows, immediately evacuated, and flushed several times with $\mathrm{He}$ or $\mathrm{H}_{2}$. The heated central $30 \mathrm{~cm}$ section of the cell was enclosed with a split-tube oven that was highly regulated and maintained at a temperature selected to obtain the desired absorption in the spectral region of interest. The cell was filled with $\mathrm{H}_{2}$ at initial pressures of 100 to 600 Torr, and the temperature was raised gradually while measurements of the line absorption were made. When the desired absorption of the region of interest was obtained, the temperature was stabilized. Typical operating temperatures were about $500 \mathrm{~K}$ to measure the line center, and up to $900 \mathrm{~K}$ to measure the weaker absorption in the line wings.

Light from a $180 \mathrm{~W}$ tungsten filament source illuminated the cold entrance window of the cell through a close-packed fiber optic bundle. Quasi-collimated by virtue of the long path from the fiber through the cell, the transmitted light was sampled at the exit window with one or more optical fibers and sent to the entrance aperture of a dispersive spectrometer. The extended fiber source provided spatial averaging that improved the signal-tonoise ratio, and the efficient detectors enabled the fiber illuminator input power to be reduced to only a few watts to avoid saturating the spectroscopic detectors. Three different spectrometers were applied in separate data runs to measure the line center at high resolution, the line wing close to the center at lesser resolution but wider coverage, and the global spectrum from the ultraviolet through the near-infrared to identify the absorption from $\mathrm{Na}_{2}$ as well as from atomic $\mathrm{Na}$ perturbed by $\mathrm{H}_{2}$.

For a low-resolution survey we used an Ocean Optics QE6500-FL spectrograph with an integral Hamamatsu S70311006 back-thinned $1024 \times 58$ pixel CCD detector. With an $f / 4$ symmetrical crossed Czerny-Turner optical system, the spectrometer provided a resolution of about $60 \AA$ full width at half maximum $(F W H M)$ covering the spectrum from 3500 to $11500 \AA$. In the region of the Na resonance doublet this was about $175 \mathrm{~cm}^{-1} F W H M$. Stray light in this region was about $0.08 \%$, and accurate absorption coefficients were determined for absorption signals in the range of $10 \%$ to $90 \%$ of the background when simple linear corrections for stray light were applied during data reduction. A spatially variable filter in front of the CCD removed higher order contributions so that, for example, a signal at $6000 \AA$ was not contaminated with second-order light from $3000 \AA$. Since the data from the CCD were summed over a column of 58 pixels, the dynamic range at each pixel was approximately $10^{6}$ detected photons. Given the short $10 \mathrm{~ms}$ exposures needed in this application, the signal-tonoise ratio due to photon shot noise was $10^{3}$. Each spectrum used in the analysis was a sum of 16 spectra, which additionally reduced noise. Spectra were taken before the oven was heated, and after it was stabilized at the working temperature. The lowtemperature spectra were the background, and the ratio of the spectrum at the working temperature to the background gave the absorption.

For high-resolution spectroscopy we used a Littrow echelle spectrograph that we developed for efficient visible and nearinfrared profile measurements of spectral lines broadened by collisions in atmospheric pressure gases. The optical system consisted of an $f / 7.3,1100 \mathrm{~mm}$ focal length, apochromatic airspaced triplet with a field flattener close to the focal plane. This lens system serves as both collimator and camera in the Littrow design, and light was introduced from 61 optical fibers, each $100 \mu \mathrm{m}$ in diameter. At the light source end the fibers were close-packed into a round bundle and were enclosed in a standard SMA connector; at the spectrograph end the fibers were in a vertical array that was focused onto a $50 \mu \mathrm{m}$ chromeon-glass slit that defined the entrance aperture of the spectrograph. A $100 \AA$ bandpass interference filter centered on $5900 \AA$ was in the optical path between the fiber array and the optical slit. The $150 \mathrm{~mm}$ diameter collimated beam was incident on a Richardson Laboratory $165 \times 320 \mathrm{~mm}, 316$ groove/mm, $63^{\circ}$ blaze, echelle reflection grating on a Zerodur substrate. When the grating was set for an angle of incidence about $57^{\circ}$ degrees from the normal, it dispersed the light into the 9th order. The diffracted light was focused back through the system onto a Kodak KAF-1001E $1024 \times 1024$ CCD with $24 \mu \mathrm{m}$ square pixels that sampled the spectrum at $0.042 \AA /$ pixel. This reciprocal dispersion was adequate to fully resolve the collisionbroadened core of the $\mathrm{Na} \mathrm{D}$ lines, with coverage in a single exposure of $43 \AA$ or $124 \mathrm{~cm}^{-1}$ around the center with a resolution of $0.24 \mathrm{~cm}^{-1}$ in samples 2 pixels wide. After exposure, the region of the detector illuminated by the fiber array was summed parallel to the slit to yield a spectrum with dynamic range of 20-bits $\left(10^{6}\right)$ and a signal-to-noise about $10^{3}$ when fully exposed.

The Littrow spectrograph could be operated at lower resolution by reversing the grating and using the opposite side of the design blaze. Although less efficient when used on the complementary groove face, the echelle grating optimized for high order worked in low order with useful efficiency when rotated $180^{\circ}$ around the grating normal. For this spectrograph, the first order provided $0.688 \AA$ /pixel with the same optics, reached $5200 \AA$ on the blue side of the $\mathrm{Na} \mathrm{D}$ lines, and gave a resolution of about $4 \mathrm{~cm}^{-1}$ in samples 2 pixels wide.

To process the observed absorption spectra, we considered a measured signal

$I(\bar{v})=I_{0}(\bar{v}) \exp (-k(\bar{v}))$

as a function of wavenumber $\bar{v}$. As long as the features of interest are broader than the resolution, and the absorbers are uniformly distributed over the optical path, the absorption coefficients found in this way may be connected to the atomic and molecular line shapes. Otherwise they represent a convolution of the instrumental response with the physical system that may reduce the apparent absorption locally. We avoided these effects 
N. F. Allard et al.: Na broadened by $\mathrm{H}_{2}$

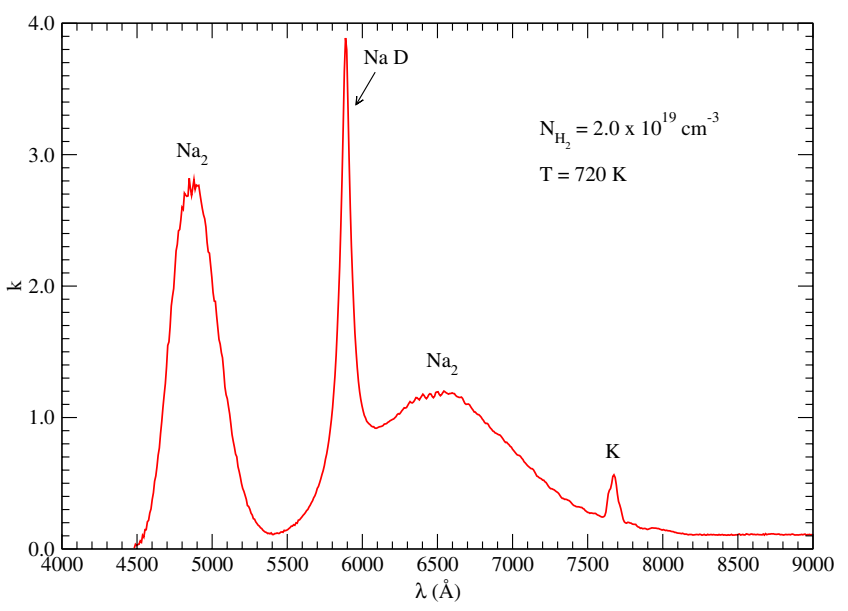

Fig. 7. Low-resolution experimental absorption coefficient $k$ of $\mathrm{Na}$ with $\mathrm{H}_{2}$ for $\left(n_{\mathrm{H}_{2}}=2.0 \times 10^{19} \mathrm{~cm}^{-3}\right.$ at $\left.720 \mathrm{~K}\right)$ over a heated cell path length of $30 \mathrm{~cm}$.

by measuring with high spectral resolution in regions with narrow features, and using low spectral resolution with caution in regions where the features are broad and change slowly with change in frequency.

The low-resolution spectrum in Fig. 7 is based on data taken with the Ocean Optics spectrograph. An initial background spectrum, recorded before Na was introduced by raising the cell temperature, is $I_{0}$. The dark zero-level signal would ideally be zero, but in actuality it is a small fraction of $I_{0}$ due primarily to light scattered inside the spectrograph.

The unresolved $\mathrm{K}$ resonance doublet appears in this spectrum because it was present as an impurity in the $\mathrm{Na}$ used for this experiment. The center of the $\mathrm{Na} \mathrm{D}$ line is more saturated than indicated here, but scattered light and low spectral resolution prevent an accurate determination of the line center with this spectrograph. However, the line wings can be extracted from the data. The contributions of the $\mathrm{Na}_{2} \mathrm{~B}-\mathrm{X}$ dimer band obscure the atomic wing for wavelengths less than $5400 \AA$, approximately $+1540 \mathrm{~cm}^{-1}$ on the blue side of the line. The $\mathrm{A}-\mathrm{X}$ dimer spectrum is also significant compared to the atomic line for wavelengths above $6100 \AA$, approximately $-580 \mathrm{~cm}^{-1}$ to the red of line center (Vdivuć et al. 2005). With an $\mathrm{X}^{1} \Sigma_{\mathrm{g}}^{+}$ground-state well depth of $0.720 \mathrm{eV}$ (Huber \& Herzberg 1979), $\mathrm{Na}_{2}$ is stable at temperatures for which the vapor pressure of atomic Na permits laboratory spectroscopy on atomic $\mathrm{Na}$. If the dimer is represented as a Gaussian it is possible to remove its effects approximately, yet unresolved structure remains and the results are only qualitative beyond these limits. A subtracted spectrum is shown in Fig. 8.

The central region of the $\mathrm{Na} \mathrm{D}$ line doublet was measured with the high-resolution spectrograph to resolve the Lorentzian core and the blending of the components with collision broadening by $\mathrm{H}_{2}$. With $\mathrm{H}_{2}$ at atmospheric pressure, the absorption coefficient of the atomic Na profiles varies by a factor of 40000 over the spectral coverage of the echelle. To measure this accurately we recorded spectra at several $\mathrm{Na}$ densities. Since the spectra overlap, the absorption coefficients determined in each instance can be connected to those for successively lower $\mathrm{Na}$ density, and a self-consistent profile can be developed that spans the region from $5875 \AA$ to $5910 \AA$. The resulting profile, normalized to unit area, is shown on a frequency or energy scale in Fig. 9.

The absorption coefficient profile may be fitted with two Lorentzian lines of unknown width, intensity ratio, and transition

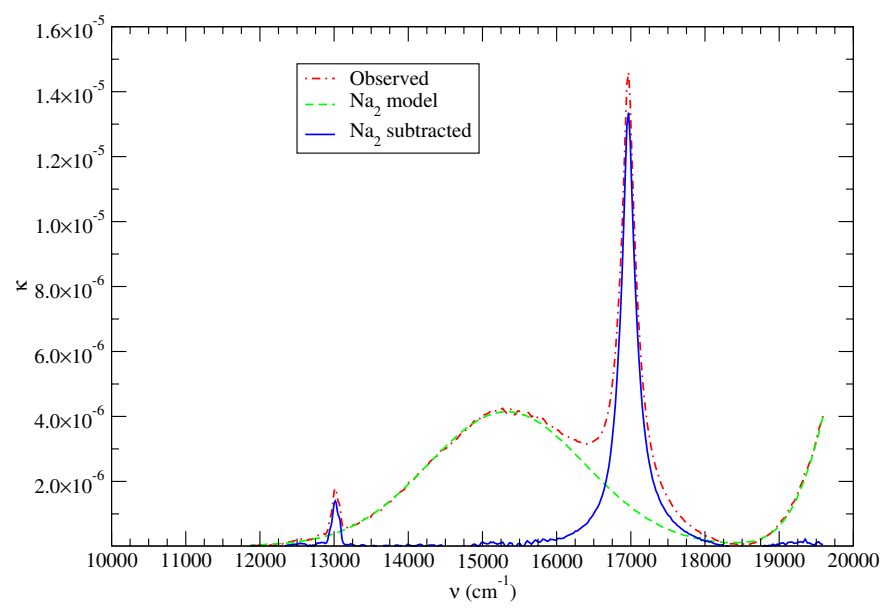

Fig. 8. Contribution of $\mathrm{Na}_{2}$ to the low-resolution experimental absorption coefficient $k$ of $\mathrm{Na}$ based on the data in Fig. 7. A small constant scattered light correction was applied, and a Gaussian model was fitted to both bands and then subtracted from the observed spectrum to yield an estimate of the atomic spectrum alone. The absorption coefficient shown is normalized to the total absorption in the atomic line, based on matching the far wing in Fig. 10.

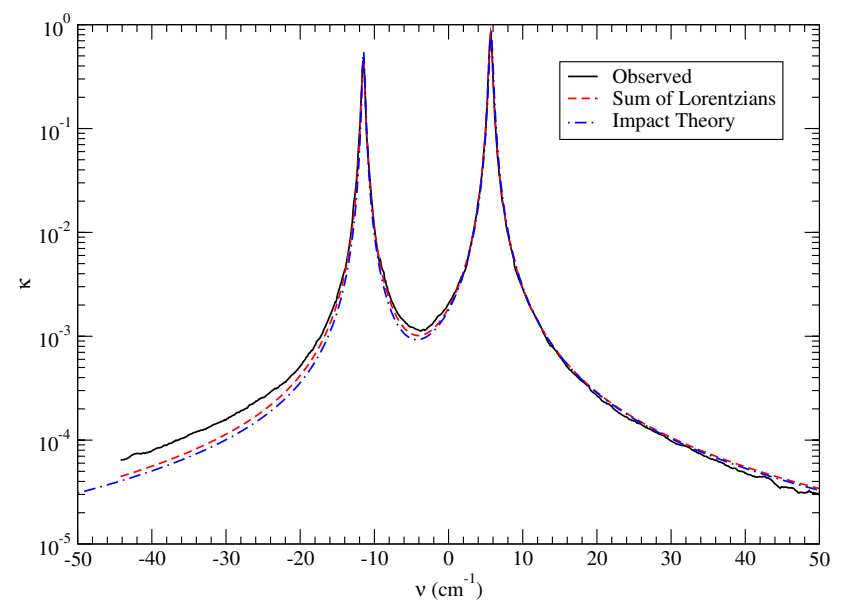

Fig. 9. Na D 3s $-3 p$ doublet line core with resolved fine structure broadened by collision with $\mathrm{H}_{2}$. This is a composite of spectra taken with cell temperatures from $470 \mathrm{~K}$ to $873 \mathrm{~K}$ joined overlapped and scaled to match. The highest temperature was used to increase the Na density and bring out the wing, and the lowest temperature was used to avoid saturation in the line center. The representative density is $n_{\mathrm{H}_{2}}=1.0 \times 10^{19} \mathrm{~cm}^{-3}$. The spectrum is shown relative to the mean $3 s-3 p$ transition energy.

energy. The result, also shown in Fig. 9, reveals that the Lorentzian behavior spans three decades of absorption coefficients from $-20 \mathrm{~cm}^{-1}$ on the red side to $+50 \mathrm{~cm}^{-1}$ or more on the blue side. The fitted ratio of areas for the components is 2.01, as expected for resonance doublets of light alkalies. The residual shifts are too small to measure. The fitted half widths at half maximum are $\gamma / n=2.26 \pm 0.04 \times 10^{-20} \mathrm{~cm}^{-2}$ for the low-frequency (D1) line, $\gamma / n=2.53 \pm 0.02 \times 10^{-20} \pm \mathrm{cm}^{-2}$ for the high-frequency (D2) line. The weight of the fitting favors large $\delta \bar{v}$, the regions of the profile measured at $873 \mathrm{~K}$.

The asymmetry of the doublet becomes apparent farther from the line. The deviation from Lorentzian behavior is real, since it connects smoothly with the large-scale line wings measured at lower resolution. On the red side, the power law fit from -20 to $-45 \mathrm{~cm}^{-1}$ is very nearly $\Delta \bar{\nu}^{-3 / 2}$. On the high-energy 


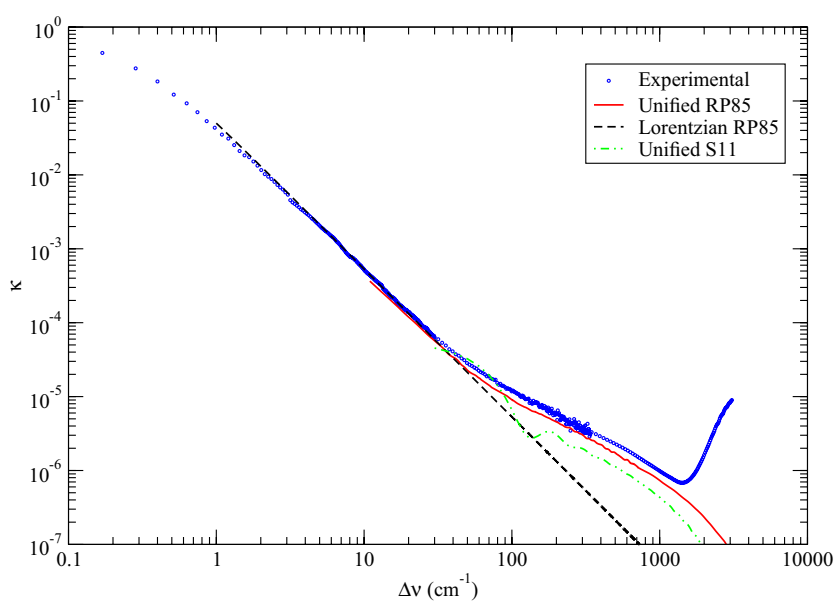

Fig. 10. Normalized absorption coefficient for the $\mathrm{Na} D 3 s^{2} \mathrm{~S}_{1 / 2}-3 \mathrm{p}^{2} \mathrm{P}_{3 / 2}$ line wing broadened by collision with $\mathrm{H}_{2}$ at $770 \mathrm{~K}$. The experimental spectrum is a composite of three segments at different resolution as described in the text. Its normalization is based on matching the core in Fig. 9 for $n_{\mathrm{H}_{2}}=1.0 \times 10^{19} \mathrm{~cm}^{-3}$.

blue side the change from Lorentzian can be attributed entirely to radiative collisions of $\mathrm{Na}$ with $\mathrm{H}_{2}$ because $\mathrm{Na}-\mathrm{Na}$ effects are comparatively small in this region. On the low-energy red side, however, the $\mathrm{Na}-\mathrm{Na}$ contributions increase significantly and it may be that the changeover to a $\Delta \bar{v}^{-3 / 2}$ power law is due in part to free-free $\mathrm{Na}-\mathrm{Na}$ collisions.

The echelle spectrograph in first order provided intermediate resolution data from the line core to the far blue wing where $\mathrm{Na}_{2}$ reappears as seen in Fig. 7. When these data are combined with the high-resolution core and the low-resolution far blue wing, we have the comprehensive measurement from the core out to more than $1000 \mathrm{~cm}^{-1}$ that is shown in Fig. 10 .

The experimental absorption coefficient $k(\bar{v})$ is a dimensionless quantity such that, for incident light of flux $I_{0}$, the transmitted flux is $I(\bar{v})$ given by Eq. (2). We define a total integrated absorption coefficient

$S=\int_{-\infty}^{+\infty} k(\bar{v}) \mathrm{d} \bar{v}$

For a fully resolved spectral line it is independent of buffer gas density in the absence of significant changes in transition probability (Kielkopf 1978). Through a column of length $\ell$ with $\mathrm{Na}$ at density $N_{\mathrm{Na}}$,

$S=\pi r_{0} f N_{\mathrm{Na}} \ell$,

where $r_{0}$ is the classical radius of the electron, and $f$ is the oscillator strength of the transition. Therefore, the normalized absorption coefficient, $\kappa(\bar{v})=k(\bar{v}) / S$, is given by

$\kappa(\bar{v})=k(\bar{v}) / \pi r_{0} f N_{\mathrm{Na}} \ell$.

It is also convenient to use a cross section $\sigma$ associated with a single absorbing atom as a measure of the absorption, in terms of which

$k(\bar{v})=\sigma(\bar{v}) N_{\mathrm{Na}} \ell$.

The normalized absorption coefficient $\kappa$ and its equivalent absorption cross section $\sigma$ do not depend on $\mathrm{Na}$ density and the absorption path length, but do depend on the density of $\mathrm{H}_{2}$ in the cell. Substituting Eq. (6) into (5) gives the relationship between the computed cross section and the normalized absorption coefficient

$\kappa(\bar{v})=\sigma(\bar{v}) / \pi r_{0} f$.
Table 1. Half-width at half maximum $\left(10^{-20} \mathrm{~cm}^{-1} / \mathrm{cm}^{-3}\right)$ of Na $3 \mathrm{~s}-3 \mathrm{p}$, $3 \mathrm{p}-4 \mathrm{~s}$ lines broadened by $\mathrm{H}_{2}$.

\begin{tabular}{lcccccc}
\hline \hline Transitions & $500 \mathrm{~K}$ & $650 \mathrm{~K}$ & $1000 \mathrm{~K}$ & $1500 \mathrm{~K}$ & $2000 \mathrm{~K}$ & $3000 \mathrm{~K}$ \\
\hline $3 \mathrm{~s}^{2} \mathrm{~S}_{1 / 2}-3 \mathrm{p}{ }^{2} \mathrm{P}_{1 / 2}$ & 1.46 & 1.6 & 1.86 & 2.16 & 2.42 & 2.8 \\
$3 \mathrm{~s}^{2} \mathrm{~S}_{1 / 2}-3 \mathrm{p}{ }^{2} \mathrm{P}_{3 / 2}$ & 1.96 & 2.16 & 2.49 & 2.94 & 3.24 & 3.87 \\
\hline $3 \mathrm{p}^{2} \mathrm{P}_{1 / 2}-4 \mathrm{~s}^{2} \mathrm{~S}_{1 / 2}$ & 4.43 & 5.07 & 5.71 & 6.67 & 7.87 & 8.89 \\
$3 \mathrm{p}^{2} \mathrm{P}_{3 / 2}-4 \mathrm{~s}^{2} \mathrm{~S}_{1 / 2}$ & 4.34 & 5.43 & 5.58 & 7.17 & 7.6 & 9.35 \\
\hline
\end{tabular}

\section{Unified profiles compared to laboratory spectra}

A unified treatment of the shape of pressure-broadened alkali absorption lines from near resonance to the far wing is obtained using an autocorrelation formalism. Complete details and the derivation of the theory are given by Allard et al. (1999). This treatment includes the finite duration of collision because it is well known that the impact approximation, which assumes that collisions occur instantaneously, causes the Lorentz theory to fail not too far from the line center.

Figures 2 and 3 of Allard et al. (2012) emphasize the nonLorentzian behavior of the two components of the $\mathrm{Na}-\mathrm{H}_{2}$ resonance line. Figure 9 of Allard et al. (2007b) presents the RP85 potential energy surfaces of $\mathrm{Na}-\mathrm{H}_{2}$ with spin-orbit coupling in the $\mathrm{C}_{2 v}$ and $\mathrm{C}_{\infty v}$ symmetry. The Lorentzian profiles were calculated using the line widths based on RP85 presented in Table 1. It is not correct to use Lorentzian profiles in the far wings (Allard et al. 2012), as is the standard practice in most stellar atmosphere work.

The experimental normalized absorption coefficients are shown in Figs. 8-10. Although the $\mathrm{Na}_{2}$ dimer is ubiquitous at laboratory temperatures, as shown in Fig. 10, its effect is less than $10^{-6}$ of the integrated line absorption in the blue wing and is largely negligible between line center and $5500 \AA$. Figure 9 shows the resolved profile of both components and the impact theory based on Table 1 . Within $10 \mathrm{~cm}^{-1}$ of the center of a component the line shape is described adequately by a Lorentzian, and the widths given in Table 1 fit the observations remarkably well for ${ }^{2} \mathrm{P}_{3 / 2}$. There is a small discrepancy for ${ }^{2} \mathrm{P}_{1 / 2}$, where the experimental width is larger than the theoretical one. The theoretical ratio of widths for $D 2 / D 1$ is $2.49 / 1.86=1.34$. We find experimentally a ratio of 1.12 .

The theoretical widths, confirmed by experiments here, are significantly greater than those predicted and measured for broadening by He, for which D2 has a HWHM of only about $1.1 \times 10^{-20} \mathrm{~cm}^{-2}$ at $500 \mathrm{~K}$ (Allard et al. 2007b; Allard \& Kielkopf, in prep.). There are no other measurements or theoretical calculations for the $\mathrm{Na}$ D-lines broadened by $\mathrm{H}_{2}$. However, the collision broadening of alkali resonance lines increases with the static polarizability of the perturber, and for noble gases is largest with $1.6 \times 10^{-20} \mathrm{~cm}^{-2}$ at $500 \mathrm{~K}$ for Xe (Kielkopf 1980). Our results for $\mathrm{H}_{2}$, which has static polarizabilities even higher than Xe, follow this trend (Huiszoon \& Briels 1993).

Figure 9 also shows that on the blue side of the line the Lorentzian shape represents the profile adequately at least to $+10 \mathrm{~cm}^{-1}(-3.5 \AA)$, and that beyond that differences between the experimental results and impact theory are not negligible. On the red side, the Lorentzian is not an adequate approximation beyond about $-5 \mathrm{~cm}^{-1}$ because the profile takes on a $\Delta \bar{v}^{-3 / 2}$ character that is sustained until the $\mathrm{Na}_{2}$ interferes with the measurement.

We can follow the far wing on the blue side out to more than $1000 \mathrm{~cm}^{-1}$ (330 ̊) before the dimer interferes. Figure 10 
shows that the Lorentzian computed from impact broadening with the RP85 potential matches the experiment close to the line, but under-represents the absorption by an order of magnitude in the far wing. There is an inflection beginning at about $+20 \mathrm{~cm}^{-1}$ in the log-log plot that is reproduced by the unified theory with the RP85 potential. The S11 potential also predicts a significant non-Lorentzian wing that is qualitatively similar to the experiment. However, the cross section predicted with S11 is less than the one observed. It does not appear that $\mathrm{Na}_{2}$ contributes significantly closer to the line than $+1100 \mathrm{~cm}^{-1}$ since in Fig. 10 the sudden onset of dimer absorption is obvious.

Figures 7 and 8 show that $\mathrm{Na}_{2} \mathrm{~B}-\mathrm{X}$ bound-bound transitions are significant in the same region, so we cannot directly detect the satellite in the laboratory. However, the profile shown in Fig. 10 clearly has started to follow the downturn predicted by the unified theory profile. This satellite is very temperaturesensitive, since it results from the potential barrier to atomic collisions in the ground state. It may be more important in exoplanet and brown dwarf atmospheres where the temperatures are higher. A similar feature in $\mathrm{K}$ has been observed in the laboratory, and in the spectrum of the brown dwarf $\varepsilon$ Indi Ba,b (Allard et al. 2007a).

\section{Conclusion}

Spectral line shapes computed in a unified theory with classical collision paths reproduce laboratory and astrophysical data. The line shape theory requires accurate atomic potentials and transition moments, which are not yet available for many systems of astrophysical interest. Laboratory observations remain a crucial tool for testing the potentials, and assumptions of line shape theory. "Satellite bands" and the shape of spectral line wings, useful in the laboratory for determining interaction potentials directly, have been seen in white dwarf and brown dwarf spectra. In previous work we presented the first applications of the absorption profiles of sodium and potassium perturbed by molecular hydrogen to the modeling of brown dwarfs and extrasolar planets (Allard et al. 2003, 2007a; Tinetti et al. 2007). Figure 1 of Allard et al. (2007a) showed a region of an exceptionally high-quality spectrum of the $\varepsilon$ Indi Ba,b. This spectrum illustrated how the visible spectra of brown dwarfs may be dominated by the strong absorption from two abundant alkalies, $\mathrm{Na}$ and $\mathrm{K}$. The abundance of $\mathrm{Na}$ being the most important, the red wing of the $\mathrm{Na}-\mathrm{H}_{2}$ profile is dominant in the overlapping region including the blue wing of $\mathrm{K}-\mathrm{H}_{2}$, and it is therefore necessary to take into account the contribution of $\mathrm{Na}$ perturbed by $\mathrm{H}_{2}$ when assessing the $\mathrm{K}$ contribution. The new $\mathrm{Na}-\mathrm{H}_{2}$ interaction potentials allow a prediction of a $\mathrm{K}-\mathrm{H}_{2}$ line satellite at the same position as in Allard et al. (2007c). This is one more theoretical confirmation that the broad absorption feature in T dwarfs, identified by Burgasser et al. (2003) as the $\mathrm{CaH}$ system is instead caused by $\mathrm{K}-\mathrm{H}_{2}$.

Figure 10 shows that the perturbation of $\mathrm{Na}$ by $\mathrm{H}_{2}$ is also very important for the interpretation of visible spectra of exoplanet atmospheres. We suggest that using Lorentzian profiles is not appropriate for modeling the far line wing, as was done, for example, in the claimed detection of TiO and VO in HD 209458b based on residuals between the models and the observations for the red wing (Désert et al. 2008). As shown in Fig. 2 of Allard et al. (2012) for the D1 component, there is an order of magnitude more absorption than an extrapolation of a Lorentzian core would predict in the region sensitive to $\mathrm{TiO}$ and $\mathrm{VO}$. As a consequence, the abundance of $\mathrm{TiO}$ and $\mathrm{VO}$ inferred from excess absorption over a Lorentzian would be too high.
In conclusion, we suggest that complete unified line profiles based on accurate atomic and molecular physics should be incorporated into analyses of exoplanet spectra when precise absorption coefficients are needed.

Acknowledgements. One of us (N.F.A.) would like to acknowledge PNPS for the financial support that allows the collaboration with the Laboratoire de Chimie et Physique Quantique in Toulouse.

\section{References}

Alioua, K., \& Bouledroua, M. 2006, Phys. Rev. A, 74, 032711

Alioua, K., Bouledroua, M., Allouche, A. R., \& Aubert-Frécon, M. 2008, J. Phys. B: At. Mol. Opt. Phys., 41, 175102

Allard, N. F. 2011a, in SF2A-2011: Proc. Annual meeting of the French Society of Astronomy and Astrophysics, eds. G. Alecian, K. Belkacem, S. Collin, R. Samadi, \& D. Valls-Gabaud, 439

http://www.sf2a.asso.fr/

Allard, N. F. 2011b, in ASP Conf. Ser. 450, eds. G. T. J. P. Beaulieu, \& S. Dieters (San Francisco: ASP), 87

Allard, N. F., \& Kielkopf, J. F. 1982, Rev. Mod. Phys., 54, 1103

Allard, N. F., \& Spiegelman, F. 2006, A\&A, 452, 351

Allard, N. F., Royer, A., Kielkopf, J. F., \& Feautrier, N. 1999, Phys. Rev. A, 60 1021

Allard, N. F., Allard, F., Hauschildt, P. H., Kielkopf, J. F., \& Machin, L. 2003, A\&A, 411, 473

Allard, N. F., Allard, F., \& Kielkopf, J. F. 2005, A\&A, 440, 1195

Allard, F., Allard, N. F., Homeier, D., et al. 2007a, A\&A, 474, L21

Allard, N. F., Kielkopf, J. F., \& Allard, F. 2007b, Eur. Phys. J. D, 44, 507

Allard, N. F., Spiegelman, F., \& Kielkopf, J. F. 2007c, A\&A, 465, 1085

Allard, N. F., Kielkopf, J. F., Spiegelman, F., Tinetti, G., \& Beaulieu, J. P. 2012, in ECLA-2011: Proc. European Conference on Laboratory Astrophysics, European Astronomical Society, eds. C. J. C. Stehlé, \& L. d'Hendecourt (Cambridge: Cambridge University Press), in press

Burgasser, A. J., Kirkpatrick, J. D., Liebert, J., \& Burrows, A. 2003, ApJ, 594, 510

Burrows, A., \& Volobuyev, M. 2003, ApJ, 583, 985

Burrows, A. M. S., Marley, M. S., \& Sharp, C. M. 2000, ApJ, 531, 438

Burrows, A., Hubbard, W. B., Lunine, J. I., \& Liebert, J. 2001, Rev. Mod. Phys., 73,719

Charbonneau, D., Brown, T. M., Noyes, R. W., \& Gilliland, R. L. 2002, ApJ, 568,377

Désert, J.-M., Vidal-Madjar, A., Lecavelier Des Etangs, A., et al. 2008, A\&A, 492,585

Foucrault, M., Millie, P., \& Daudey, J. P. 1992, J. Chem. Phys., 96, 1257

Huber, K. P., \& Herzberg, G. 1979, Molecular Spectra and Molecular Structure, IV. Constants of Diatomic Molecules (New York: Van Nostrand Reinhold Company), 432

Huiszoon, C., \& Briels, W. J. 1993, Chem. Phys. Lett., 203, 49

Huron, B., Malrieu, J. P., \& Rancurel, P. 1973, J. Chem. Phys., 58, 5745

Jensen, A. G., Redfield, S., Endl, M., et al. 2011, ApJ, 743, 203

Kielkopf, J. 1978, J. Phys. B At. Mol. Opt. Phys., 11, 25

Kielkopf, J. F. 1980, J. Phys. B At. Mol. Opt. Phys., 13, 3813

Kielkopf, J. F. 1983, J. Phys. B At. Mol. Opt. Phys., 16, 3149

Liebert, J., Reid, I., Burrows, A., et al. 2000, ApJ, 533, L155

Müller, W., Flesch, J., \& Meyer, W. 1984, J. Chem. Phys., 80, 3297

Redfield, S., M. Endl, W. D. C., \& Koesterke, L. 2008, ApJ, 673, L87

Rossi, F., \& Pascale, J. 1985, Phys. Rev. A, 32, 2657

Sing, D. K., Vidal-Madjar, A., Lecavelier des Etangs, A., et al. 2008, ApJ, 686, 667

Snellen, I. A., Albretch, S., Mooij, E. J. W., \& Poole, R. S. L. 2008, A\&A, 487, 357

Snellen, I. A. G., de Kok, R. J., de Mooij, E. J. W., \& Albrecht, S. 2010, Nature, 465, 1049

Spiegelmann, F., \& Malrieu, J. P. 1984, J. Phys. B At. Mol. Phys., 17, 1259

Swain, M. R., Vasisht, G., \& Tinetti, G. 2008, Nature, 452, 329

Swain, M. R., Deroo, P., Griffith, C. A., et al. 2010, Nature, 463, 637

Szudy, J., \& Baylis, W. 1975, J. Quant. Spectr. Rad. Transf., 15, 641

Szudy, J., \& Baylis, W. 1996, Phys. Rep., 266, 127

Tinetti, G., Vidal-Madjar, A., Liang, M.-C., et al. 2007, Nature, 448, 169

Vdivuć, S., Beuc, R., Aumiler, D., Ban, T., \& Pichler, G. 2005, J. Phys. B At. Mol. Opt. Phys., 38, 3107

Waldmann, I. P., Tinetti, G., Drossart, P., et al. 2012, ApJ, 744, 35

Zhu, C., Babb, J. F., \& Dalgarno, A. 2005, Phys. Rev. A, 71, 052710

Zhu, C., Babb, J. F., \& Dalgarno, A. 2006, Phys. Rev. A, 73, 012506 